\title{
Criminologie
}

\section{Le traitement correctionnel fondé sur des données probantes : une recension}

\section{Franca Cortoni et Denis Lafortune}

Volume 42, numéro 1, printemps-été 2009

L’intervention à l'aune des données " probantes »

URI : https://id.erudit.org/iderudit/029808ar

DOI : https://doi.org/10.7202/029808ar

Aller au sommaire du numéro

\section{Éditeur(s)}

Les Presses de l'Université de Montréal

ISSN

0316-0041 (imprimé)

1492-1367 (numérique)

Découvrir la revue

Citer cet article

Cortoni, F. \& Lafortune, D. (2009). Le traitement correctionnel fondé sur des données probantes : une recension. Criminologie, 42(1), 61-89.

https://doi.org/10.7202/029808ar
Résumé de l'article

Contrairement à la perspective « Nothing works » de Martinson (1974), l'approche « What works ? » du traitement correctionnel s'est centrée sur la possibilité que le traitement correctionnel soit efficace dans la réduction de la récidive criminelle des délinquants. Les preuves empiriques examinées dans le présent article corroborent le fait que l'application des principes risque-besoins-réceptivité d'Andrews et Bonta (2006) donnent les bases d'un modèle efficace de réhabilitation. Pourtant, malgré les grands progrès réalisés dans le développement et la mise en oeuvre d'un traitement correctionnel fondé sur des données probantes, il subsiste des sphères qui nécessitent un approfondissement. Dans cet article, trois questions souvent négligées relativement à la réhabilitation du délinquant sont aussi examinées, à savoir la nécessité de prendre en considération les enjeux motivationnels chez les délinquants, l'importance des compétences et attitudes du personnel, et la nécessité de documenter et d'évaluer continuellement les pratiques de réhabilitation. 


\title{
Le traitement correctionnel fondé sur des données probantes: une recension
}

\author{
Franca Cortoni \\ Professeure adjointe \\ École de criminologie, Université de Montréal \\ franca.cortoni@umontreal.ca \\ Denis Lafortune \\ Professeur agrégé \\ École de criminologie, Université de Montréal \\ denis.lafortune@umontreal.ca
}

RÉSUMÉ - Contrairement à la perspective «Nothing works» de Martinson (1974), l'approche "What works?» du traitement correctionnel s'est centrée sur la possibilité que le traitement correctionnel soit efficace dans la réduction de la récidive criminelle des délinquants. Les preuves empiriques examinées dans le présent article corroborent le fait que l'application des principes risque-besoins-réceptivité d'Andrews et Bonta (2006) donnent les bases d'un modèle efficace de réhabilitation. Pourtant, malgré les grands progrès réalisés dans le développement et la mise en œuvre d'un traitement correctionnel fondé sur des données probantes, il subsiste des sphères qui nécessitent un approfondissement. Dans cet article, trois questions souvent négligées relativement à la réhabilitation du délinquant sont aussi examinées, à savoir la nécessité de prendre en considération les enjeux motivationnels chez les délinquants, l'importance des compétences et attitudes du personnel, et la nécessité de documenter et d'évaluer continuellement les pratiques de réhabilitation.

MOTS-CLÉS - Traitement correctionnel, préparation au changement, caractéristiques des intervenants, données probantes.

\section{Introduction}

Le traitement correctionnel des délinquants a pour objectif général de gérer et réduire les risques de récidive criminelle (Lösel, 2001 ; Andrews

Traduit de l'anglais par Grégoire Dodier et Pierre Drolet. 
et Bonta, 2006; Hollin, 2006). Malheureusement, il peut arriver que cet objectif soit interprété comme s'il signifiait que le traitement n'est alors axé que sur les lacunes des délinquants et qu'il utilise de façon mécanique des techniques et des méthodes conçues pour réduire et éliminer des comportements indésirables ou inadaptés, ainsi que les cognitions qui leur sont associées (Hollin, 2006). Il ne faut pas se surprendre que le traitement conçu pour les délinquants soit critiqué et qu'on lui reproche de ne pas prendre en considération les caractéristiques individuelles des délinquants, ni les besoins psychosociaux "au sens large» qu'ils pourraient avoir en tant qu'êtres humains (Quirion, 2006; Ward et Maruna, 2007). Ce type d'argument est en partie fondé sur un point de vue qui veut que le traitement correctionnel soit réducteur et qu'il considère le délinquant comme un ensemble de «déficits» à combler, au lieu de prendre en compte la personne dans sa globalité.

Par ailleurs, il ne faut pas oublier que des enjeux légaux et éthiques limitent le type de traitement qu'il est possible de conduire auprès des délinquants: bien que les cliniciens qui travaillent dans le système de justice pénale soient autorisés à traiter les comportements criminels, ils ne sont pas habilités pour autant à s'occuper des sphères de la vie des délinquants qui sont sans lien avec le comportement criminel (ex.: la Loi sur le système correctionnel et la mise en liberté sous condition [Canada, 1992]; voir aussi Ogloff, 1995 et McGuire, 2004). Cela dit, un traitement conçu pour s'attaquer aux comportements criminels ne doit-il pas viser à améliorer en même temps la qualité de vie des délinquants? De fait, les approches correctionnelles qui réduisent efficacement la récidive sont des interventions positives, constructives et centrées sur l'acquisition d'habiletés qui visent les problèmes à la source du comportement criminel, le développement de nouvelles compétences et la mise en valeur des forces de l'individu, tout cela dans le but d'aider celui-ci à mener une vie saine et sans délinquance (McGuire, 2004; Ward et Maruna, 2007).

Bien qu'historiquement on ait pu croire que «rien ne fonctionne» («Nothing works») lorsque vient le temps de modifier le comportement criminel (Martinson, 1974), un grand nombre de données empiriques récentes montrent qu'en matière de traitement correctionnel, au contraire, quelques approches spécifiques peuvent être efficaces (Cullen et Gendreau, 1989; Andrews et Bonta, 2006). Andrews et ses collègues ont proposé puis intensivement mis à l'épreuve trois principes-clés sur 
lesquels appuyer les «services à la personne ${ }^{1} »$, principes qui sont maintenant connus sous le nom de risque-besoins-réceptivité. À l'origine, leur travail a été grandement aiguillonné par la conclusion de Martinson selon laquelle rien n'est censé fonctionner en matière de réduction de la récidive. Aujourd'hui, il existe un vaste corpus de données empiriques qui montrent qu'un traitement basé sur les principes du risque, des besoins et de la réceptivité mène à de plus importantes réductions des taux de récidive que les seules sanctions de la justice pénale (Hollin et Palmer, 2006b). Cependant, pour que le traitement correctionnel soit une réussite, ces auteurs tout comme d'autres (ex. : Bernfeld et al., 2001; Hollin et Palmer, 2006a) soulignent comment, outre l'application des trois principes-clés, les problèmes d'implantation du traitement, le besoin de documenter et d'évaluer les efforts de réhabilitation sont des facteurs importants qui doivent être pris en considération. Chacune de ces conditions de réussite est au cœur de cet article.

\section{Principes d'intervention correctionnelle efficace}

Toute discussion du traitement correctionnel efficace présuppose une bonne compréhension des prémisses du modèle de réhabilitation d'Andrews et Bonta (2006). À partir d'une théorie du comportement criminel basée sur la personnalité et sur les processus cognitifs de l'apprentissage social, Andrews et Bonta (2006) ont développé puis raffiné au cours des deux dernières décennies des principes d'intervention correctionnelle efficace. Bien que techniquement Andrews (2001) ait examiné un total de 18 principes spécifiques, trois s'avèrent prépondérants, i. e. le risque, les besoins et la réceptivité. Ils regroupent un certain nombre de principes plus spécifiques qui traitent directement $\mathrm{du}$ «qui, quoi et comment» en matière de traitement auprès des délinquants. Quant aux autres principes, ils sont liés plus précisément au processus général de développement et d'implantation des interventions, deux problèmes qui seront examinés plus loin dans cet article.

1. Dans un contexte correctionnel, la notion de «services à la personne» désigne l'ensemble des interventions thérapeutiques destinées aux délinquants. Elles se distinguent des sanctions légales (ex.: amendes, probation, emprisonnement) et des interventions qui se concentrent peu sur les caractéristiques individuelles des délinquants, telles que les «boot camps» ou camps d'entraînement et les programmes dissuasifs de type «scared-straight». 


\section{Intervenir auprès de qui ?}

La sélection des participants pour le traitement correctionnel se fait normalement en fonction du principe du risque. Dans ce contexte, le traitement ne se fonde pas sur un modèle médical selon lequel le comportement criminel est une psychopathologie. La notion de traitement désigne plutôt un ensemble d'interventions psychosociales ${ }^{2}$ conçues pour réduire les risques de récidive (Hollin, 2006). Le principe du risque détermine l'intensité du traitement qu'un délinquant devrait recevoir. De façon plus précise, il stipule que les délinquants les plus à risque ont davantage besoin d'interventions/de suivi et, qu' inversement, les délinquants à faible risque n'ont besoin que de peu ou pas d'interventions. Tel qu'il est énoncé par Andrews et Dowden (2006: 89), on peut se dire que: "si ce n'est pas brisé, il est inutile de le réparer». Demander à des délinquants présentant de faibles risques de participer à un traitement intensif n'améliorera aucunement l'ensemble des résultats (Andrews et Dowden, 2006). De la même façon, un traitement insuffisant ne changera en rien les taux de récidive chez les délinquants à risque élevé. À titre d'illustration, rappelons que dans leur étude portant sur le traitement de 482 délinquants incarcérés, Bourgon et Armstrong (2005) trouvent que chez les délinquants à haut risque qui ont bénéficié d'au moins 300 heures de traitement, les taux de récidive sont significativement moindres $(38,3 \%)$ que ceux observés auprès des délinquants qui présentent des risques comparables mais n'ont reçu que 100 heures de traitement (62\%).

Au fond, il y a deux éléments constitutifs du principe du risque: évaluer le risque et le faire correspondre à l'intensité du traitement proposé (Andrews et al., 1990; Andrews et al., 2006). Pour évaluer le risque, il est nécessaire de bien comprendre les facteurs spécifiquement associés au comportement criminel. De manière générale, pour évaluer les risques de récidive, l'exercice du jugement professionnel ou clinique non structuré produit des prédictions qui n'ont que peu de valeur, c'està-dire qui font à peine mieux que le hasard. Le problème tient au fait que ces jugements «traditionnels» prennent souvent en compte des facteurs qui ne sont pas nécessairement liés à la récidive (Andrews et al., 2006; Quinsey et al., 2006; Quinsey, 2008). Par exemple, la recherche a pu démontrer que les troubles mentaux et les problèmes

2. Voilà pourquoi les termes «traitement» et «intervention» sont utilisés de manière interchangeable dans le présent article. 
psychologiques importants tels que l'anxiété et la dépression n'ont aucun rapport avec les risques de récidive (Bonta et al., 1998; Hanson et Bussière, 1998; Hanson et Morton-Bourgon, 2005; Quinsey et al., 2006). D'autres facteurs traditionnellement jugés importants, tels que la présence de remords, le degré de force utilisée dans l'infraction, la gravité des blessures subies par la victime ou le degré d'intrusion du comportement criminel dans les cas d'agressions sexuelles (ex.: des rapports sexuels complets comparés à des attouchements), n'ont pas de rapport avec les risques de récidive (Hanson et Bussière, 1998; Quinsey et al., 2006). Dans l'évaluation du risque, il faut considérer uniquement les caractéristiques individuelles et particulières du délinquant qui augmentent ou diminuent la probabilité d'une récidive. Une telle compréhension des «facteurs de risque» est fondée sur des données empiriques qui font la démonstration d'un rapport entre ces caractéristiques et une récidive éventuelle (Hanson et Morton-Bourgon, 2005; Andrews et Bonta, 2006; Andrews et al., 2006; Quinsey et al., 2006).

Dans le modèle d'Andrews, il existe deux types de facteurs de risque: statiques et dynamiques. Les facteurs de risque statiques peuvent être définis comme des aspects du passé du délinquant qui sont liés à la récidive, mais ne peuvent être changés au moyen d'interventions. Les antécédents criminels et l'âge sont des exemples de tels facteurs statiques. Quant aux facteurs dynamiques, ils désignent les caractéristiques du délinquant qui peuvent être changées et qui, une fois amendées, sont liées à un changement dans les taux de récidive (Andrews et al., 1990; Andrews et Bonta, 2006; Hanson, 1997). Les attitudes antisociales, la fréquentation de personnes antisociales ou une faible maîtrise de soi sont des exemples de facteurs dynamiques. Ajoutons que l'évaluation du risque de récidive est mieux effectuée lorsqu'on utilise des instruments dont la validité prédictive, dans le contexte qui nous intéresse, a été démontrée (Hanson, 1997; Andrews et al., 2006; Quinsey et al., 2006).

Les délinquants, comme tous les êtres humains, présentent des différences individuelles qui influent sur leurs chances de s'engager dans une large gamme de comportements. Par conséquent, ils vont aussi différer les uns des autres quant à leurs chances de s'engager dans un nouveau comportement criminel. Voilà pourquoi l'évaluation valide du risque est une première étape qui offre la possibilité de différencier les délinquants pour ensuite les apparier au niveau de traitement qui est le plus approprié pour chacun. Cet appariement assure que les délinquants reçoivent 
l'intensité d'intervention qui correspond à leur niveau de risque. En procédant de la sorte, les probabilités que l'intervention réussisse sont augmentées (Andrews et Bonta, 2006).

Il s'installe parfois une confusion entre la sévérité de la sanction pénale et la notion de risque. En effet, il peut être présumé que les sanctions les plus lourdes (ex. : les sentences les plus longues) dénotent un risque de récidive plus élevé et que, par conséquent, des interventions plus intensives sont nécessaires. L'inverse est également vrai: un délinquant ayant reçu une sanction plus légère est souvent perçu comme s'il présentait un risque plus faible de récidive et qu'il ne nécessitait que très peu d'interventions. Or, la recherche a démontré que le risque de récidive n'est pas associé à la sévérité des sanctions (Andrews et Dowden, 2006). Cela n'est guère étonnant, dans la mesure où les principes de justice pénale qui guident l'imposition de sanctions ne sont pas basés sur des estimations du risque de récidive, mais plutôt sur la nature et la gravité du comportement criminel ${ }^{3}$. Par contre, le risque de récidive est bel et bien associé à l'effet du traitement. Les délinquants qui présentent un risque élevé de récidive (peu importe leur sanction officielle) bénéficient plus du traitement que les délinquants à faible risque, mais seulement lorsque ce traitement respecte le principe des besoins et celui de la réceptivité (Andrews et Dowden, 2006).

\section{Pour traiter quels problèmes (quoi ?)}

Le principe des besoins suggère que les besoins criminogènes, par opposition aux besoins psychologiques plus généraux, sont les cibles de traitement appropriées. Les besoins criminogènes représentent les éléments directement liés au comportement criminel et qui sont modifiables. Il existe un vaste et riche corpus théorique, soutenu par des données empiriques, à propos des facteurs liés au comportement criminel en général, mais aussi à certains types spécifiques de délits comme les crimes violents et les crimes sexuels (ex.: Polaschek et Coolie, 2004; Andrews et Bonta, 2006; Ward et al., 2006; Gannon et al., 2007). Dans tous ces travaux, il est reconnu que le comportement criminel est appris

3. On trouve deux exceptions dans le Code criminel du Canada: les désignations de «délinquant à contrôler» et "délinquant dangereux», qui sont fondées sur des évaluations des risques futurs (Greenspan et Rosenberg, 2007). 
plutôt qu'inné, et que de nombreux facteurs liés à la criminalité sont modifiables grâce à l'intervention correctionnelle.

Les besoins criminogènes sont également appelés facteurs criminogènes ou facteurs de risque dynamiques. Il s'agit en fait des mêmes facteurs qui devraient faire partie d'une évaluation complète du risque (Andrews et al., 2006). On remarque qu'il y a présentement, dans ce champ d'intervention, un débat quant à savoir si les «besoins » criminogènes sont considérés par les délinquants eux-mêmes comme des «besoins» éprouvés (ou des problèmes vécus), qui nécessitent des interventions (McGuire, 2004; Ward et Maruna, 2007). À titre d'exemple, rappelons que certains délinquants peuvent considérer l'usage de la violence comme un élément essentiel dans leur vie; et qu'il est très peu probable qu'ils considèrent que leur point de vue sur la violence est un «besoin» sur lequel on devrait intervenir. Par conséquent, parler des besoins criminogènes en termes de facteurs criminogènes ou de facteurs liés au comportement criminel risque d'être une option plus acceptable pour les délinquants (et même pour les intervenants). L'utilisation d'un tel vocable pourrait également aider les délinquants à saisir la relation qui s'établit entre ces problèmes et leur comportement criminel, ce qui pourrait les aider à comprendre la rationalité du traitement correctionnel.

Indépendamment de ces questions de sémantique, la recherche montre que les facteurs criminogènes sont d'une grande pertinence clinique lorsque le but d'une intervention consiste à gérer et réduire les risques d'un plus grand engagement dans le comportement criminel (Andrews et Bonta, 2006). Le ciblage de facteurs psychologiques "généraux» ou de facteurs spécifiques, mais qui n'ont aucun rapport avec le comportement criminel, une proposition qui est habituellement séduisante pour bon nombre de cliniciens en santé mentale (Ogloff et Davis, 2004), n'entraîne malheureusement pas une réduction des risques de récidive (Andrews et al., 1990; Andrews et Bonta, 2006). Lorsque les facteurs criminogènes sont ciblés de manière appropriée, on constate une amélioration considérable des résultats (c'est-à-dire une réduction de la récidive; McGuire, 2001b); tandis que l'inclusion de cibles inappropriées risque de réduire l'efficacité du traitement (Andrews et Bonta, 2006).

Cela dit, se concentrer sur des facteurs criminogènes ne signifie pas qu'il faille appliquer d'une manière générale des interventions qui s'avéreraient identiques pour tous. Les facteurs criminogènes, bien que globalement pertinents pour tous les délinquants, vont se manifester 
différemment d'un individu à un autre. Par conséquent, un délinquant ne devrait pas automatiquement recevoir un traitement pour contrer des facteurs qui, bien que pertinents pour la plupart des délinquants, ne sont pas présents chez lui. Au contraire, le traitement devrait être toujours basé sur une formulation individualisée des facteurs criminogènes (Andrews et Bonta, 2006; Hollin, 2006). Par ailleurs, on sait que les interventions qui sont axées sur une combinaison de facteurs criminogènes réduisent plus efficacement la récidive criminelle que celles qui se concentrent sur un seul facteur (Goggin et Gendreau, 2006). Les données probantes indiquent que l'efficacité est accrue lorsqu'on tient compte simultanément d'au moins trois facteurs criminogènes différents (French et Gendreau, 2006). Par exemple, l'efficacité de l'intervention sera augmentée si, plutôt que de cibler simplement la gestion de la colère chez les délinquants violents ${ }^{4}$, le traitement vise aussi les cognitions (attitudes et croyances) et les relations interpersonnelles problématiques qui favorisent l'utilisation de la violence.

Bien qu'il y ait plusieurs points communs entre les divers facteurs criminogènes, quelques-uns sont spécifiques à certaines sous-populations de délinquants (ex. : les hommes qui se livrent à de la violence familiale ou les délinquants sexuels). Par conséquent, une connaissance approfondie des travaux empiriques traitant des facteurs criminogènes spécifiques à certains types de délinquants paraît nécessaire pour qu'un traitement efficace puisse être dispensé. Les facteurs criminogènes comprennent: des attitudes antisociales générales; les attitudes liées à certains types spécifiques de crimes tels que la violence ou les agressions sexuelles; les déficits dans le traitement des informations sociales; le (manque de) contrôle de la colère; une faible maitrise de soi; les modes de vie problématiques et instables (ex.: le manque d'éducation ou de compétences professionnelles; le rejet des valeurs sociales liées à l'éducation et au travail); les relations familiales et interpersonnelles instables; les associations avec des personnes antisociales; l'abus d'alcool ou d'autres drogues (Andrews et Bonta, 2006; McGuire, 2006). Les délinquants sexuels présentent des caractéristiques additionnelles et uniques qui comprennent la déviance sexuelle et les préoccupations sexuelles (Hanson et Morton-Bourgon, 2005).

4. Ceci n'est utilisé qu'à titre d'exemple, car tous les délinquants violents n'ont pas nécessairement des problèmes de contrôle de la colère (Polaschek, 2006). 


\section{Avec quel type d'intervention (comment?)}

Autant les interventions correctionnelles doivent se fonder sur un cadre théorique solide qui permet d'analyser les causes du comportement criminel, autant le choix des modalités de traitement appliquées aux délinquants devrait être basé sur des données empiriques. Le principe de la réceptivité est celui qui aborde le mieux cet enjeu: la sélection des modes et des styles de traitement devrait être basée sur des données probantes qui spécifient quel type de traitement fonctionne généralement bien auprès des délinquants, par comparaison avec les populations non délinquantes. Le principe de la réceptivité spécifie aussi que l'intervention devrait prendre en considération les caractéristiques individuelles qui peuvent influer sur la capacité de chacun à bénéficier du traitement (Andrews et Bonta, 2006).

Auprès des délinquants, les traitements dont l'efficacité a été empiriquement démontrée sont essentiellement des interventions structurées de type cognitivo-comportementale (Lipton et al., 2001 ; Lösel, 2001 ; Landenberger et Lipsey, 2005; Lösel et Schmucker, 2005; Andrews et Bonta, 2006). À ce jour, les thérapies non structurées, non directives, d'inspiration psychodynamique ou orientées sur l'introspection ne se sont pas révélées efficaces pour réduire le comportement criminel (Andrews et al., 1990; Lösel et Schmucker, 2005; Andrews et Bonta, 2006). En fait, les délinquants affichent des caractéristiques, telles que des problèmes d'impulsivité, de pauvres capacités de résolution des problèmes, des attitudes antisociales et une faible motivation pour le traitement, qui les empêchent de s'engager significativement dans les interventions non directives et orientées sur l'introspection (voir Andrews et al., 1990; Wong, 2000; Wong et Hare, 2005; Andrews et Bonta, 2006; Day et al., 2006). En général, les interventions considérées efficaces incluent: la restructuration cognitive; le modelage et le renforcement des comportements anticriminels; l'acquisition graduelle d'habiletés diverses; le renforcement et le jeu de rôle pour consolider ces nouvelles habiletés; l'apport des ressources/de soutien; et la formulation de suggestions concrètes (ex. : souligner le besoin de changer ou inciter à faire certains choix) (Andrews et Bonta, 2006).

On tend à négliger les caractéristiques individuelles des délinquants lorsque vient le moment de mettre en place un traitement correctionnel. Pourtant, les styles cognitifs, les traits de personnalité, les styles et capacités d'apprentissage, ainsi que des facteurs affectifs tels que la 
présence d'anxiété, une faible estime de soi ou un inconfort psychologique généralisé peuvent influer sur les capacités des délinquants à bénéficier d'un traitement (Andrews et Bonta, 2006). L'âge, le genre et les origines ethnoculturelles sont d'autres facteurs (statiques, cette fois) de réceptivité, qui devraient être pris en considération. En somme, les délinquants devraient avoir la capacité de s'engager significativement dans un traitement (Ward, Howells et Birgden, 2004). On devrait donc considérer que les facteurs de réceptivité sont une partie intégrante du traitement correctionnel. Cela dit, il est important de noter qu'ils ne peuvent en rien le remplacer puisqu'un traitement ciblé principalement ou exclusivement sur des facteurs non criminogènes n'influerait pas sur la récidive (Andrews et Dowden, 2007).

Outre les aspects qui peuvent faire obstacle au traitement, les aspects positifs devraient être identifiés et renforcés. Indépendamment de leurs besoins criminogènes, les délinquants ont invariablement des forces qu'il faudrait mettre à profit durant le traitement. Accroître les forces présentes, tout en tenant compte des facteurs criminogènes, permet de brosser un portrait plus complet du délinquant et de proposer des avenues pour l'établissement d'objectifs orientés vers un avenir positif qui soit irréconciliable avec le crime (Andrews et Dowden, 2007; Ward et Maruna, 2007). Enfin, la motivation des délinquants face au traitement est un facteur de réceptivité important auquel on devrait accorder un grand intérêt. Comme nous en discuterons plus loin, la motivation à entreprendre le traitement devrait faire l'objet d'une attention particulière.

L'efficacité d'une application de ces principes de traitement correctionnel a été confirmée par la recherche, particulièrement par l'utilisation des méta-analyses. Rappelons que la méta-analyse est une méthode statistique qui permet la mise en commun des résultats de nombreuses études individuelles. Ce faisant, elle vise à contrôler statistiquement les variations et les biais potentiels observés d'une étude à l'autre pour ensuite déterminer si, globalement, le traitement a un impact réel. Les méta-analyses permettent de tirer une conclusion sur «l'ampleur de l'effet». Cette statistique, dans le cas des variables continues, peut être interprétée comme une différence moyenne (exprimée en pourcentage) mesurée entre les résultats des groupes traités et non traités.

À ce jour, plus de 50 méta-analyses ont examiné l'efficacité des principes risque-besoins-réceptivité lorsqu'ils sont appliqués auprès des délinquants adultes et juvéniles, des femmes délinquantes, des délinquants provenant de minorités ethniques, des délinquants violents et 
des délinquants sexuels (Hollin et Palmer, 2006b). Les résultats de ces méta-analyses montrent que lorsque le traitement correctionnel adhère aux principes risque-besoins-réceptivité, des réductions de la récidive sont invariablement observées. L'ampleur de l'effet se situe généralement entre 0,10 et 0,30 , bien que des valeurs plus grandes de l'ordre de 0,25 à 0,35 soient plus courantes (Andrews et Bonta, 2006; Goggin et Gendreau, 2006). À ce point-ci de l'exposé, un exemple pourrait aider à comprendre la façon d'interpréter l'ampleur de l'effet: des taux de récidive de $30 \%$ (TT $=$ taux des traités) mesurés chez des délinquants traités, comparativement à des taux de $45 \%$ (TNT = taux des non traités) pour des délinquants équivalents, mais non traités, auraient pour résultat une ampleur de l'effet de 0,15. Cette différence mesurée entre les délinquants traités et non traités peut également signifier que le traitement a mené à une réduction de la récidive de $33 \%$ (calculée de cette façon: [TT - TNT]/TNT; dans notre exemple $[30-45] / 45=33 \%$ ).

En termes de signification clinique, il importe de souligner que les ampleurs de l'effet évaluées par les méta-analyses du traitement correctionnel sont tout à fait comparables, voire dans quelques cas supérieures, à celles d'interventions médicales bien crédibles telles que l'usage d'aspirine et du pontage coronarien pour réduire le risque de crise cardiaque (Lipton, 1992, 1995; McGuire, 2001a; Marshall et McGuire, 2003). De plus, des analyses d'efficience montrent que chaque dollar investi dans des interventions correctionnelles efficaces génère un retour qui se situe entre $1,13 \$$ et $8,44 \$$ (Welsh et Farrington, 2001; Conference Board of Canada, 2009). Ce montant est fort appréciable, compte tenu du fait que les coûts d'administration de la justice pénale ne cessent d'augmenter. Plus importante encore, la réduction des taux de récidive signifie également qu'il y a moins de victimes éventuelles, moins de coûts pour le système de justice pénale et des contributions sociétales positives accrues de la part des ex-délinquants. Cette économie des «coûts indirects» n'est pas toujours incluse dans les calculs

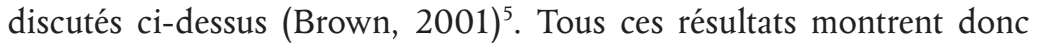
l'importance d'adhérer aux principes risque-besoins-réceptivité lorsqu'on élabore et implante des interventions correctionnelles (Ogloff et Davis, 2004; Hollin et Palmer, 2006b). En fait, même les détracteurs du modèle risque-besoins-réceptivité reconnaissent qu'une preuve

5. Le Conference Board of Canada (2009) a inclus des estimations de ces coûts dans ses calculs. 
empirique impressionnante témoigne de son efficacité à réduire les taux de récidive (McGuire, 2004; Ward et Maruna, 2007).

\section{L'implantation de traitements correctionnels efficaces}

Le problème de la motivation et de l'abandon du traitement

L'abandon du traitement est un phénomène très répandu, dont l'importance est bien reconnue dans les travaux qui portent sur le traitement correctionnel. Des études montrent que les délinquants qui entreprennent mais qui ne complètent pas le traitement ont des taux de récidive plus élevés que ceux qui terminent le traitement ou que ceux qui n'en commencent aucun (Hanson et Bussière, 1998; Wormith et Olver, 2002). Généralement, les délinquants abandonnent le traitement parce qu'il ne répond pas à leurs attentes, souvent erronées. Par exemple, ils jugent le traitement trop exigeant ou ils ne croient pas qu'il puisse être pertinent (Day et al., 2006). De plus, certains délinquants peuvent avoir été retirés du programme en raison de leurs comportements perturbateurs ou de leur manque d'assiduité. Une autre cause potentielle d'abandon est le manque de correspondance entre le programme indiqué et celui qui est réellement offert. Par exemple, si un délinquant est évalué comme ayant un moindre besoin d'intervention dans une sphère donnée et qu'il doit, néanmoins, entreprendre un programme destiné aux délinquants ayant des besoins plus élevés, il pourrait se décourager et décrocher.

Les motifs d'abandon du traitement sont souvent considérés comme une indication du manque de motivation de la part du délinquant. Ils peuvent être liés à une réticence à s'engager dans le traitement, autrement dit à une résistance (Preston, 2001); à des difficultés à envisager les bénéfices inhérents au traitement (Wild et al., 1998); ou à une pauvre qualité de l'alliance de travail avec les intervenants (Proulx et al., 2004). Dans un cas comme dans l'autre, il y a lieu de parler d'un manque de préparation au traitement (Howells et Day, 2003; Ward, Howells et Birgden, 2004).

Bien que préliminaire, la recherche en ce domaine commence à indiquer qu'il faut porter une attention particulière aux problèmes de motivation vécus par les délinquants. Dans son étude des facteurs liés à une réduction de la récidive après la fin du traitement chez les 
délinquants sexuels, Marchand (2008) a trouvé qu'une plus grande motivation et une meilleure collaboration pendant le traitement caractérisaient les délinquants sexuels traités et non récidivistes. Par ailleurs, Nunes et Cortoni (2006), après avoir examiné les variables qui peuvent prédire l'abandon de toute forme de traitement correctionnel, ont trouvé qu'une faible motivation à entreprendre un traitement était liée à une augmentation du risque d'abandon. La motivation est donc une variable importante qui doit être prise en considération dans les milieux correctionnels.

Tout traitement correctionnel gagnerait à inclure des éléments conçus pour évaluer, augmenter et maintenir la motivation avant, pendant et après le traitement. Avant le traitement, certains délinquants devraient bénéficier de services centrés sur la motivation (Nunes et Cortoni, 2006). Il existe des modèles de préparation au changement et des stratégies d'entretiens motivationnels, communément utilisés dans les centres d'intervention en matière de consommation de drogues et d'alcool, qui fournissent des suggestions concrètes sur la façon d'aider les délinquants à développer leur motivation et à prendre conscience des problèmes causés par le comportement criminel (Prochaska et DiClemente, 1983 ; Miller et Rollnick, 1991 ; Day et al., 2006). En cours de traitement, les délinquants devraient recevoir des encouragements pour souligner les progrès accomplis (Fernandez et Serran, 2002). Dans ce contexte, il est tout aussi indiqué de les amener à analyser périodiquement leurs propres progrès afin de favoriser chez eux la prise de conscience et la compréhension des gains réalisés. Quand ils progressent bien, les délinquants tendent à ne pas le remarquer, tandis qu'ils excellent dans l'art de se concentrer sur leurs difficultés (Cortoni et Carich, 2007). La capacité de se concentrer sur des événements positifs, et non pas seulement sur les difficultés, permet de déployer des efforts plus soutenus durant les périodes difficiles (Langston, 1994; Folkman et Moskowitz, 2000). Prendre soin d'aider les délinquants à développer cette capacité durant le traitement augmente la possibilité qu'une fois le traitement terminé, ils maintiennent leurs efforts pour tendre vers une vie prosociale et sans crime. Dans cette perspective, Ward et Maruna (2007) développent depuis quelques années un modèle dit « de bonnes vies» («good lives model»), qui met un accent particulier sur les objectifs personnels du délinquant et sur l'application des principes de la psychologie positive. En quelques mots, ce modèle en est un qui postule que lorsqu'on aide les individus à atteindre des «biens humains» (ex.: 
la santé, l'autonomie ou la connaissance) par des moyens non criminels, il est possible d'éliminer ou de réduire leur besoin de recourir à la délinquance.

\section{L'importance de la compétence des intervenants}

Les caractéristiques des intervenants sont des ingrédients importants dans tout effort de réhabilitation réussie. L'efficacité du traitement correctionnel est accrue lorsque les services sont dispensés par des intervenants qui appuient les objectifs et les stratégies du traitement et qui, en même temps, peuvent servir de modèles «anticriminels», tout en renforçant les attitudes ou les efforts prosociaux des délinquants (Dowden et Andrews, 2004; Andrews et Bonta, 2006). Certaines attitudes affichées par les intervenants ont démontré leur valeur positive indépendamment du type de traitement, d'environnement correctionnel ou de délinquants. Elles incluent les dispositions à : communiquer avec les délinquants de façon claire, ouverte, attentive, enthousiaste et respectueuse; aider les délinquants à faire la distinction entre une règle et une demande; démontrer et renforcer des solutions de rechange «vivantes» (i. e. incarnées par l'intervenant) aux façons de penser, de sentir et d'agir qui favorisent la délinquance; fournir un environnement thérapeutique positif et structuré; aider les délinquants à déterminer et à surmonter les obstacles qui les empêchent de s'engager dans des comportements prosociaux (Andrews et Bonta, 2006).

Il n'est pas étonnant que l'authenticité, la capacité de ne pas juger, le respect, la chaleur et l'empathie, des caractéristiques qui contribuent à une relation thérapeutique positive peu importe le type de client, prennent une importance tout aussi grande lorsqu'on travaille avec des délinquants (Andrews, 1983). Cela dit, il est important de ne pas confondre l'acceptation et l'empathie envers le délinquant, d'une part, et l'acceptation inconditionnelle des fausses perceptions qu'il a de luimême, des autres et de son délit, d'autre part. En fait, l'acceptation inconditionnelle est contreproductive lorsqu'on travaille avec une population criminelle puisqu'elle ne fait que renforcer, et non pas réduire, les facteurs qui contribuent au comportement criminel (Andrews et Bonta, 2006). D'autres comportements ou attitudes facilitent le traitement: adopter une approche socratique plutôt que didactique, poser des questions ouvertes, être flexible, encourager et récompenser la participation, donner de l'espoir et de la confiance, et être émotion- 
nellement sensibles aux clients (Fernandez et Serran, 2002). Ces comportements aident à établir un climat thérapeutique positif pendant le traitement et la présence d'un tel climat contribue à son tour à l'efficacité du traitement pour réduire la récidive (Dowden et Andrews, 2004 ; Beech et Hamilton-Giachritsis, 2005).

De façon générale, peu importe le niveau de compétence interpersonnelle et clinique des intervenants, ceux-ci devraient être engagés dans leur mission de réhabilitation et bien formés à la dispensation des traitements correctionnels (McGuire, 2001a). Les intervenants devraient comprendre non seulement les principes de l'intervention cognitivocomportementale, mais aussi les fondements théoriques et empiriques qui expliquent le comportement criminel (Andrews, 2001). En plus de posséder des qualifications pertinentes, ils devraient avoir accès à de la formation continue et à de la supervision clinique offerte par des superviseurs compétents en matière d'interventions correctionnelles, mais aussi dans le contenu spécifique du traitement (ex. : violence conjugale; Andrews, 2001; Dowden et Andrews, 2004; Goggin et Gendreau, 2006). La formation et la supervision devraient aussi se centrer sur les facteurs de réceptivité de manière à aider les intervenants à dispenser des interventions complexes d'une façon qui puisse faire du sens pour les délinquants (Hollin et Palmer, 2006b). Enfin, les organismes devraient s'assurer que les intervenants disposent des ressources nécessaires pour bien effectuer leur travail. Comme McGuire (2001a) l'a déclaré: «aucun traitement connu ni matériel de formation ne permet à lui seul d'atteindre les objectifs poursuivis en l'absence d'intervenants formés, engagés dans leur mission, pourvus des ressources suffisantes et qui peuvent compter sur le soutien de la direction» (2001a: 34).

\section{Évaluation du traitement correctionnel}

Il est devenu inacceptable d'élaborer et d'implanter des traitements correctionnels sans, par la même occasion, préparer et mettre en place un cadre d'évaluation (McGuire, 2001b; Hollin, 2006). La réalisation de recherches évaluatives visant à déterminer l'efficacité du traitement correctionnel est un élément important, bien que souvent négligé, des interventions correctionnelles. Trop souvent, la conception d'une recherche en matière de traitement correctionnel reste une activité reportée à un avenir lointain, une fois que le traitement aura été appliqué pendant plusieurs années, qu'un temps suffisant se sera écoulé et 
qu'on aura pu mesurer les taux de récidive. Il arrive même que la recherche évaluative soit menée seulement pour répondre aux demandes de cadres supérieurs et de décideurs qui commencent à mettre en doute l'utilité des interventions correctionnelles, notamment à la lumière des coûts qu'ils engendrent (Brown, 2001; Welsh et Farrington, 2001).

En fait, la conception d'un cadre d'évaluation visant à vérifier l'efficacité du traitement correctionnel doit se faire à chaque stade du développement de l'intervention et ce cadre doit être revu et modifié au fur et à mesure que des changements sont apportés au programme (Patton, 1997). Cet exercice de rigueur permet que des mesures de protection soient mises en place pour prévenir les erreurs et permettre que les conclusions tirées soient valides (McGuire, 2001b; Hollin, 2006). Omettre de concevoir et de mettre en place un cadre d'évaluation durant l'élaboration du traitement produira des résultats très insatisfaisants, puisque des questions auxquelles il est impossible de répondre seront inévitablement soulevées.

\section{Un modèle logique}

Il existe un grand nombre d'objectifs évaluatifs qui peuvent être regroupés en deux grandes catégories: l'évaluation formative ou de processus et l'évaluation sommative ou de l'efficacité. L'évaluation de processus est menée pour apprécier les problèmes liés à l'implantation et au suivi de l'intervention, tandis que l'évaluation de l'efficacité détermine si oui ou non les résultats escomptés ont été atteints (McGuire, 2001b). À chaque stade du développement de l'intervention, un devis de recherche devrait être établi pour valider à la fois le processus de traitement et les résultats escomptés, tout en s'assurant que ces résultats ont bel et bien été obtenus grâce au programme. Le devis de recherche doit, par conséquent, garantir que les résultats attendus sont bien définis et opérationnalisés. Plus encore, il faut être certain que, dans le devis de recherche, diverses variables sont contrôlées de façon à ce qu'on puisse clairement attribuer le changement au programme.

Une fois élaboré, un modèle «logique» offre un cadre utile pour faire le lien entre les objectifs et les résultats escomptés d'un programme (Porteous et al., 2002; Ogborne, 2003). Il constitue aussi une bonne source d'information pour déterminer quelles sont les autres variables, telles que la durée de l'incarcération ou la participation à d'autres programmes, qui pourraient être liées aux résultats (McGuire, 2001b). 
Contrôler ces variables est une étape cruciale pour qui veut être en mesure de démontrer qu'un résultat est dû précisément à l'intervention évaluée et non pas à d'autres raisons. Un modèle logique de type «organigramme» établit les liens entre les principales composantes du programme, les objectifs d'implantation et les résultats souhaités à court et à long terme. Il permet de planifier des évaluations axées sur la prise de décisions. Les questions et les méthodes de recherche découlent naturellement du modèle et les activités associées à la recherche peuvent alors être clarifiées. Il s'agit notamment de déterminer qui utilisera les résultats de la recherche et à quelles fins, d'estimer les ressources à court et à long terme nécessaires pour mener la recherche et d'établir les méthodes de collecte des données (Porteous et al., 2002; Ogborne, 2003). Le modèle sert également de base pour identifier les évaluations de résultats intermédiaires et à long terme. Enfin, il permet de faire en sorte que tous les aspects liés à la conception, l'implantation et l'évaluation du programme soient précisés d'avance. En d'autres termes, l'utilisation d'un modèle logique comme étape initiale pour concevoir un cadre d'évaluation aide à clarifier les objectifs, à cerner les éléments manquants, à distinguer la fin et les moyens, à faire en sorte que les résultats escomptés soient clairement liés aux composantes du programme, et à corriger les suppositions erronées à propos de ce que le programme est censé réaliser.

L'évaluation de processus et l'évaluation de l'efficacité sont normalement incluses dans le cadre plus large. Une évaluation de processus vise à établir dans quelle mesure l'intervention correctionnelle fonctionne bien et comment elle peut être améliorée. Une évaluation de l'efficacité vise à déterminer si l'intervention correctionnelle réussit à aborder les facteurs criminogènes et si une modification de ces facteurs est associée aux résultats escomptés, tels que l'abstinence ou la diminution de l'abus des substances, une meilleure gestion de soi ou une réduction de la récidive.

\section{Les évaluations de processus}

L'objectif général des évaluations de processus est d'examiner les problèmes liés au bon déroulement de l'intervention. De telles évaluations fournissent des informations portant sur l'intégrité du traitement correctionnel tel qu'il a été implanté, de même que sur les problèmes liés à la gestion du traitement et de ses utilisateurs (Andrews et Dowden, 
2005). Ces évaluations sont requises pour examiner la qualité générale de l'implantation et de la dispensation du traitement (Leschied et al., 2001). Il s'agit également de savoir si le programme répond aux attentes des intervenants, des gestionnaires et des délinquants eux-mêmes.

Une évaluation de processus examine généralement les questions suivantes: Est-ce que les bons participants ont été engagés dans le programme? Comment ces participants ont-ils réagi au traitement (ex. : l'engagement; la cohésion du groupe; une participation active; la satisfaction par rapport au processus; la satisfaction par rapport au matériel abordé dans le traitement; la qualité de la relation avec les dispensateurs de traitement; les taux d'abandon; les motifs d'abandon)? Est-ce que le traitement a été dispensé d'une manière conforme aux normes de l'établissement (ex.: le cadre; les compétences; la formation; la supervision clinique des intervenants) ? Est-ce que le traitement a été dispensé tel que théoriquement prévu (ex.: les modalités et le contenu du traitement sont donnés en conformité avec le manuel de référence)? Est-ce que l'environnement général est favorable au traitement (ex. : les autres membres du personnel ont pris connaissance du programme et l'appuient; des informations collatérales sur le comportement des délinquants sont disponibles; les rapports rédigés en cours ou à la fin du traitement sont utiles aux agents de libération conditionnelle et autres décideurs; la direction apporte son soutien et s'assure que les ressources appropriées sont allouées au traitement)? Finalement est-ce que le programme tient compte des problèmes de réceptivité (ex.: le niveau d'alphabétisation, les problèmes de santé mentale ou la motivation)? Ces questions ne constituent pas une liste exhaustive des éléments du processus qui peuvent être examinés lors d'une évaluation formative. Elles n'ont pour but que d'indiquer quels sont les aspects importants qui permettent de s'assurer que le programme de traitement est implanté et dispensé en conformité avec le plan initial et qu'il répond bel et bien aux besoins des délinquants (Gendreau et al., 1999; Andrews, 2001).

\section{Les évaluations de l'efficacité}

L'objectif général des évaluations de l'efficacité est de déterminer si l'intervention a atteint ses objectifs et permis d'obtenir les résultats escomptés. Les dits «résultats» sont des changements qui devraient découler de la participation à l'intervention. Ce type d'évaluation s'intéresse donc à ce qui a changé à la suite du traitement. Les change- 
ments devraient apparaître à court et à long terme et être en lien avec cet objectif général qu'est la réduction des risques de récidive. Un résultat à court terme est défini comme un produit immédiat qui peut être attendu de la participation à l'intervention correctionnelle. Passer d'une attitude procriminelle à une attitude prosociale ou faire preuve d'une meilleure capacité à gérer des problèmes d'impulsivité ou de colère sont des exemples de tels résultats à court terme. Des questions de recherche spécifiques et des hypothèses vérifiables au sujet de l'efficacité de l'intervention s'appuieront sur les résultats escomptés. De telles questions et hypothèses devraient être définies durant la phase de développement de l'intervention, afin que des procédures appropriées de collecte de données soient établies et permettent éventuellement une mise à l'épreuve.

Les résultats à long terme peuvent être définis comme les changements attendus qui se manifesteraient d'eux-mêmes une fois le traitement complété depuis un certain temps. Dans ce contexte, des changements de comportements tels qu'une réduction des comportements perturbateurs pendant l'incarcération, une amélioration de la stabilité d'emploi lorsque la personne vit en communauté ou une réduction de la récidive sont des exemples de résultats à long terme. Les questions de recherche typiques sont alors: Est-ce que l'intervention a réussi à apporter les changements cognitifs et comportementaux escomptés chez les participants? Est-ce que ces changements se sont maintenus au fil du temps? Est-ce que l'intervention et les changements qui en résultent sont liés à une réduction de la récidive?

Dans les évaluations de l'efficacité, des comparaisons planifiées entre les délinquants traités et non traités font invariablement partie du devis d'évaluation. Comme dans n'importe quelle recherche tentant d'établir l'impact d'un traitement, un devis de recherche avec assignation aléatoire des sujets est jugé le meilleur pour déterminer si le traitement correctionnel a eu un impact sur les taux des nouvelles condamnations (Hollin, 2006). Dans un tel devis, les délinquants ayant des caractéristiques comparables en termes de risque et de facteurs criminogènes sont répartis aléatoirement entre le groupe de traitement et le groupe témoin. Les comparaisons vérifient si : a) grâce à l'intervention, des changements sur le plan des facteurs criminogènes se sont produits dans le groupe traité; b) les taux de récidive sont plus bas chez les sujets du groupe traité; c) les changements observés à la suite du traitement sont associés à des taux de récidive réduits. 
Cela dit, en général, l'essai clinique avec assignation aléatoire est très difficile à implanter dans un environnement correctionnel. Une solution de rechange consiste à utiliser des devis quasi expérimentaux, les sujets non traités du groupe témoin étant appariés aux sujets traités du groupe expérimental pour des variables-clés qui risquent d'être associées aux résultats (Hollin, 2006). Minimalement, les variables d'appariement importantes devraient comprendre: l'âge, le niveau de risque global présenté par le délinquant et les besoins criminogènes qui ont été identifiés. D’autres aspects tels que les contextes, le moment et la durée de l'incarcération devraient aussi être considérés. Par «appariement des contextes», il faut ici entendre les conditions dans lesquelles se retrouvent les délinquants traités et non traités, les derniers devant provenir du même type d'environnement correctionnel que ceux qui ont été exposés au programme (Losël, 2001). Par exemple, il faudrait comparer des délinquants traités incarcérés à des délinquants non traités incarcérés et des délinquants traités suivis dans la communauté à des délinquants non traités suivis dans la communauté. Une autre problématique importante à considérer lorsqu'on crée des groupes de comparaison est de savoir si les délinquants ont participé à des interventions autres que celle qui est à l'étude. Toute évaluation de l'impact d'un traitement spécifique nécessite qu'on tienne compte de la participation possible à d'autres interventions correctionnelles. Tel que Losël (2001) l'a fait remarquer, plus l'environnement carcéral est «thérapeutique», plus il est difficile d'établir l'efficacité d'une intervention particulière. Cette situation montre bien l'importance de créer des groupes de comparaison appropriés et, lorsque nécessaire, d'utiliser des méthodes statistiques pour contrôler l'effet des différences prétraitement entre les groupes ou l'effet d'une participation à d'autres traitements correctionnels (Dowden et Serin, 2001 ; Cortoni et al., 2006).

\section{Conclusion}

Bien que la recherche ait clairement démontré que le traitement correctionnel peut être efficace pour réduire la récidive, il est encore très fréquent qu'on omette d'appliquer les principes qui contribuent à cette efficacité. Bien qu'ils ne soient pas très récents, les travaux de Gendreau et al. (2001) ont analysé les résultats de trois vastes recensions des interventions correctionnelles au Canada et aux États-Unis. Elles incluaient 291 programmes différents. Au total, Gendreau et al. (2001) 
ont trouvé que $70 \%$ de ces programmes correctionnels n'avaient pas respecté les principes d'un traitement correctionnel efficace. Les problèmes les plus courants étaient: des intervenants insuffisamment formés, à qui les travaux théoriques et empiriques portant sur le comportement criminel n'étaient pas familiers; des procédures d'évaluation du risque inexistantes ou des procédures qui n'étaient pas à la hauteur des normes professionnelles actuelles en matière d'évaluation du risque; la prestation de services centrés sur des facteurs non criminogènes ou l'utilisation d'approches qui se sont avérées inefficaces pour traiter les facteurs criminogènes; l'omission presque complète de tenir compte des facteurs de réceptivité des délinquants dans le traitement; et enfin un manque d'évaluations (de processus ou d'impact) systématiques. L'ensemble de ces problèmes indique que les systèmes correctionnels ont encore du mal à implanter des interventions et des programmes efficaces. Ces difficultés peuvent être dues à un manque de connaissances, à une pénurie de ressources, ou sinon, à une simple résistance aux interventions correctionnelles basées sur les principes d'Andrews et Bonta (2006).

Le problème du manque de connaissances pourrait être facilement corrigé grâce à la formation et au transfert des connaissances. Au minimum, on devrait exiger que les intervenants en milieu correctionnel possèdent des connaissances suffisantes dans un domaine pertinent comme la criminologie ou la psychologie. L'éducation universitaire a toutefois ses limites, surtout si les intervenants ne s'assurent pas de maintenir leurs connaissances professionnelles à jour. Étant donné l'augmentation rapide des données probantes sur les facteurs de risque et les interventions auprès des délinquants (ex.: Hanson et al., 2007), un transfert périodique des connaissances assuré par des spécialistes pourrait s'avérer une stratégie rentable pour les systèmes correctionnels dans la mesure où cela permettrait aux intervenants de maintenir leurs connaissances à jour quant aux avancées théoriques et empiriques les plus récentes. Cette formation ne pourra être efficace, toutefois, que si elle est véritablement soutenue (et même dans certains cas imposée) par la direction.

Le manque de ressources représente un problème beaucoup plus difficile à résoudre. Les systèmes correctionnels coûtent cher (ex.: le Rapport du SCC sur les plans et les priorités 2007-2008 [Canada, 2007]), et leurs ressources sont généralement attribuées par des organismes gouvernementaux. Dans ce contexte, et en dépit des meilleures intentions, les gestionnaires de services correctionnels risquent de ne pas 
avoir l'autorité suffisante pour allouer un financement approprié au traitement des délinquants. Une argumentation militant en faveur d'un niveau approprié de soutien financier devrait s'adresser aux organismes subventionnaires avec l'objectif de leur faire comprendre la rentabilité des interventions correctionnelles lorsqu'on les compare à l'incarcération et à ses coûts. Malheureusement, en dépit des meilleurs efforts déployés par les gestionnaires des services correctionnels, des augmentations de subventions pour les interventions correctionnelles sont trop souvent accordées après que des délits importants (ex.: agressions sexuelles, meurtres) ont été commis par des délinquants en libération conditionnelle qui avaient été mal évalués et gérés. Bien que de toute évidence aucun système correctionnel ne puisse prévenir totalement de tels événements malheureux, les données probantes pèsent lourdement en faveur de l'évaluation du risque et de la gestion clinique des délinquants, y compris de la prestation des services d'intervention appropriés. En s'assurant que leurs pratiques tiennent compte de ces données, les systèmes correctionnels seront mieux équipés pour gérer différemment les délinquants à haut risque, tout en s'assurant que les délinquants à faible risque ne sont pas soumis inutilement à des pratiques sévères (Andrews et Dowden, 2007).

La résistance aux interventions correctionnelles basées sur les principes risque-besoins-réceptivité constitue, au fond, le problème le plus difficile à résoudre. Quelques systèmes correctionnels (ou quelques individus dans ces systèmes) peuvent choisir de rejeter tout simplement les données probantes en matière d'interventions efficaces, que ce soit par opportunisme ou en raison de décisions prises par des gestionnaires, des établissements ou des groupes sociopolitiques. Dans d'autres cas, les intervenants peuvent avoir des orientations théoriques divergentes qui n'incluent pas les approches cognitivo-comportementales, qui ne sont pas axées sur les facteurs de risque dynamiques ou qui préfèrent privilégier des modèles sociologiques ou psychopathologiques du crime comme base théorique pour guider l'intervention correctionnelle. C'est en de telles circonstances que la pratique fondée sur des données probantes devient cruciale. Les intervenants devraient se demander si la pratique qu'ils ont choisie est corroborée par la recherche. Cela ne ferait aucun sens, quelles que soient les orientations théoriques, institutionnelles ou sociopolitiques choisies, de dispenser des interventions dont l'inefficacité a été démontrée (Goggin et Gendreau, 2006). De plus, on pourrait alléguer que fournir délibérément des services inefficaces est 
contraire aux règles de l'éthique et en violation avec le mandat des systèmes correctionnels, qui considèrent la réduction de la récidive comme un objectif.

Bien que des controverses subsistent dans le champ de la réhabilitation des délinquants (Ward et Maruna, 2007), il est difficile de s'imaginer que dans les systèmes correctionnels sont implantées volontairement des pratiques d'intervention dont l'inefficacité est démontrée. Cela dit, malgré la disponibilité de données probantes, beaucoup de travail reste à faire pour consolider les résultats prometteurs et développer la capacité d'intervenir plus efficacement dans le domaine de la réhabilitation des délinquants (Andrews et Dowden, 2007). La poursuite des recherches pour améliorer notre compréhension des pratiques correctionnelles efficaces est donc une nécessité.

\section{Références}

Andrews, D. A. (1983). Assessment of outcomes in correctional samples. In M. L. Lambert, E. R. Christensen \& S. S. DeJulio (eds.), The Measurement of Psychotherapy Outcome in Research and Evaluation (160-201). New York: John Wiley \& Sons.

Andrews, D. A. (2001). Principles of effective correctional programs. In L. L. Motiuk \& R. C. Serin (eds.), Compendium 2000 on Effective Corrections (I, 9-17). Ottawa: Correctional Service Canada.

Andrews, D. A., \& Bonta J. (2006). The psychology of criminal conduct (4 $4^{\text {th }}$ Ed.). Cincinnati: Anderson.

Andrews, D. A., Bonta, J., \& Hoge, R. D. (1990). Classification for effective rehabilitation. Criminal Justice and Behavior, 17, 19-52.

Andrews, D. A., Bonta, J., \& Wormith, J. S. (2006). The recent past and near future of risk and/or need assessment. Crime $\mathcal{O}$ Delinquency, 52, 7-27.

Andrews, D. A., \& Dowden, C. (2005). Managing correctional treatment for reduced recidivism: A meta-analytic review of program integrity. Legal and Criminological Psychology, 10, 173-187.

Andrews, D.A., \& Dowden, C. (2006). Risk principle of case classification in correctional treatment: A meta-analytic investigation. International Journal of Offender Therapy and Comparative Criminology, 50, 88-100.

Andrews, D.A., \& Dowden, C. (2007). The Risk-Need-Responsivity model of assessment and human service in prevention and corrections: Crime-prevention jurisprudence. Canadian Journal of Criminology and Criminal Justice, 49, 439-464.

Andrews, D. A., Zinger, I., Hoge, R. D., Bonta, J., Gendreau, P., \& Cullen, F. T. (1990). Does correctional treatment work? A clinically relevant and psychologically informed meta-analysis. Criminology, 28, 369-404. 
Beech, A. R., \& Hamilton-Giachritsis, C. E. (2005). Relationship between therapeutic climate and treatment outcome in group-based sexual offender treatment programs. Sexual Abuse: A Journal of Research and Treatment, 17, 127-140.

Bernfeld, G. A., Farrington, D. P., \& Leschied, A. W. (Eds.) (2001). Offender rehabilitation in practice: Implementing and evaluating effective programs. Chichester, UK: John Wiley \& Sons.

Birgden, A. (2004). Therapeutic jurisprudence and responsivity: Finding the will and the way in offender rehabilitation. Psychology, Crime and Law, 10, 283-295.

Bonta, J., Law, M., \& Hanson, R. K. (1998). The prediction of criminal and violent recidivism among mentally disordered offenders: A meta-analysis. Canadian Journal of Criminology, 39, 127-144.

Bourgon, G. \& Armstrong, B. A. (2005). Transferring the principles of effective treatment into a "real world" prison setting. Criminal Justice and Behavior, 32, 3-25.

Brown, S. L. (2001). Cost-effective correctional treatment. In L. L. Motiuk \& R. C. Serin (eds.), Compendium 2000 on Effective Corrections (I, 221-233). Ottawa: Correctional Service Canada.

Canada (1992). Loi sur le système correctionnel et la mise en liberté sous condition. Ottawa: Département de Justice, Gouvernement du Canada.

Canada (2007) Rapport sur les plans et les priorités du Service correctionnel du Canada 2007-2008. Ottawa : Service correctionnel du Canada. Disponible sur www. csc-scc.gc.ca

Cortoni, F., \& Carich, M. S. (2007). The how and why of building coping skills in treatment. In D. Prescott (ed.), Applying knowledge to practice: Challenges in the treatment and supervision of sexual abusers (222-238). Oklahoma City, OK: Wood'N'Barnes.

Cortoni, F., Nunes, K. L., \& Latendresse, M. (2006). An examination of the effectiveness of the Violence Prevention Program. Research Report R-178. Ottawa: Correctional Service Canada.

Cullen, F. T., \& Gendreau, P. (1989). The effectiveness of correctional rehabilitation - reconsidering the "nothing works" debate. In L. Goodstein \& D. L. McKenzie (eds.), The American prison: Issues in research and policy (23-44). New York: Plenum.

Day, A., Bryan, J., Davey, L., \& Casey, S. (2006). The process of change in offender rehabilitation programmes. Psychology, Crime, N Law, 12, 473487.

Dowden, C. \& Andrews, D. A. (2004). The importance of staff practice in delivering effective correctional treatment: A meta-analytic review of core correctional practice. International Journal of Offender Therapy and Comparative Criminology, 48, 203-214.

Dowden, C., \& Serin, R. (2001). Anger management Programming for Offenders: The impact of program performance measures. Research Report $R-106$. Ottawa: Correctional Service Canada. 
Fernandez, Y. M., \& Serran, G. (2002). Characteristics of an effective sex offender therapist. In B. Schwartz (ed.), The Sex offender vol. 4: Current treatment modalities and systems issues (9.1-9.12). Kingston, NJ: Civic Research Institute.

Folkman, S., \& Moskowitz, J. T. (2000). Positive affect and the other side of coping. American Psychologist, 55, 647-654.

French, S. A., \& Gendreau, P. (2006). Reducing prison misconducts: What works! Criminal Justice and Behavior, 33, 185-218.

Gannon, T. A., Ward, T., Beech, A. R., \& Fisher, D. (2007). Aggressive Offenders' Cognition: Theory, Research and Practice. Chichester, England: John Wiley \& Sons.

Gendreau, P., Goggin, C., \& Smith, P. (1999). The forgotten issue in effective correctional treatment: program implementation. International Journal of Offender Therapy and Comparative Criminology, 43, 180-187.

Gendreau, P., Goggin, C., \& Smith, P. (2001). Implementation guidelines for correctional programs in the 'real world'. In G. A. Bernfeld, D. P. Farrington \& A. W. Leschied (eds.), Offender Rebabilitation in Practice: Implementing and evaluating effective programs (247-268). Chichester, UK: Wiley.

Goggin, C., \& Gendreau, P. (2006). The implementation and maintenance of quality services in offender rehabilitation programmes. In C. R. Hollin, \& E. J. Palmer (eds), Offending behaviour programmes: Development, application, and controversies (209-246). Chichester, UK: Wiley \& Sons.

Greenspan, E. L., \& Rosenberg, M. (2007). Martin's annual criminal code. Aurora, ON : Canada Law Book Inc.

Hanson, R. K. (1997). Sex offender risk assessment. In C. R. Hollin (ed.), Handbook of Offender Assessment and Treatment (85-96). Chichester, UK: Wiley.

Hanson, R. K., \& Bussière, M. T. (1998). Predicting Relapse: A meta-analysis of sexual offender recidivism studies. Journal of Consulting and Clinical Psychology, 66, 348-362.

Hanson, R. K., Harris, A. J. R., Scott, T. L., \& Helmus, T. (2007). Assessing the risk of sexual offenders on community supervision: The Dynamic Supervision Project. (User Report No. 2007-05). Ottawa: Corrections Research, Public Safety Canada.

Hanson, R. K., \& Morton-Bourgon, K. E. (2005). The characteristics of persistent sexual offenders: A meta-analysis of recidivism studies. Journal of Consulting and Clinical Psychology, 73, 1154-1163.

Hollin, C. R. (2006). Offending behaviour programmes and contention: Evidence-based practice, manuals, and programme evaluation. In C. R. Hollin, $\&$ E. J. Palmer (eds), Offending behaviour programmes: Development, application, and controversies (33-67). Chichester, UK: Wiley \& Sons.

Hollin, C. R., \& Palmer, E. J. (Eds.) (2006a). Offending behaviour programmes: Development, application, and controversies. Chichester, UK: Wiley \& Sons.

Hollin, C. R., \& Palmer, E. J. (2006b). Offending behaviour programmes: Controversies and resolutions. In C. R. Hollin \& E. J. Palmer (eds.), Offending 
behaviour programmes: Development, application, and controversies (247-278). Chichester, UK: Wiley \& Sons.

Howells, K., \& Day, A. (2003). Readiness for anger management: Clinical and theoretical issues. Australian and New Zealand Journal of Psychiatry, Psychology, and Law, 11, 189-196.

Langston, C. A. (1994). Capitalizing on and coping with daily-life events: Expressive responses to positive events. Journal of Personality and Social Psychology, 67, 1112-1125.

Leschied, A. W., Bernfeld, G. A., \& Farrington, D. P. (2001). Implementation issues. In G. A. Bernfeld, D. P. Farrington \& A. W. Leschied (eds.), Offender Rebabilitation in Practice: Implementing and evaluating effective programs (3-19). Chichester, UK: Wiley.

Lipsey, M. W. (1992). Juvenile delinquency treatment: A meta-analytic inquiry into the variability of effects. In T. D. Cook, H. Cooper, D. S. Cordray, H. Hartmann, L. V. Hedges, R. J. Light, T. A. Louis \& F. Mosteller (eds.) Metaanalysis for explanation: A Casebook (83-127) New York: Sage.

Lipsey, M. W. (1995). What do we learn from 400 research studies on the effectiveness of treatment with juvenile delinquents? In J. McGuire (ed.), What Works: Reducing Reoffending - Guidelines From Research and Practice (6378). UK: Wiley.

Lipton, D. S., Pearson, F. S., Cleland, C. M., \& Yee, D. (2001). The effectiveness of cognitive- behavioural treatment methods on offender recidivism : Metaanalytic outcomes from the CDATE project. In J. McGuire (ed.), Offender Rebabilitation and Treatment: Effective Programmes and Policies to reduce Reoffending (79-112). UK: Wiley \& Sons.

Lösel, F. (2001). Evaluating the effectiveness of correctional programs: Bridging the gap between research and practice. In G. A. Bernfeld \& D. P. Farrington (eds.), Offender Rehabilitation in Practice (67-92). Chichester, UK: Wiley.

Lösel, F., \& Schmucker, M. (2005). The effectiveness of treatment for sexual offenders: A comprehensive meta-analysis. Journal of Experimental Crimino$\log y, 1,117-146$.

Marchand, A. (2008). L'implication dans le traitement et la récidive des agresseurs sexuels adultes. Mémoire de maîtrise, Université de Montréal, Montréal.

Marshall, W. L., \& McGuire, J. (2003). Effect sizes in the treatment of sexual offenders. International Journal of Offender Therapy and Comparative Crimino$\log y, 47,653-663$.

Martinson, R. (1974). What Works? Questions and answers about prison reform. The Public Interest, 36, 22-54.

McCulloch, A., \& McMurran, M. (2007). The features of a good offender treatment programme manual: A Dephi survey of experts. Psychology, Crime ๙v Law, 13, 265-274.

McGuire, J. (2001a). What works in correctional intervention? Evidence and practical implications. In G. A. Bernfeld, D. P. Farrington \& A. W. Leschied (eds.), Offender Rebabilitation in Practice: Implementing and evaluating effective programs (25-43). Chichester, UK: Wiley. 
McGuire, J. (2001b). Development of a program Logic Model to assist evaluation. In L. L. Motiuk, \& R. C. Serin (eds.), Compendium 2000 on Effective Corrections, $(I, 208-220)$. Ottawa: Correctional Service Canada.

McGuire, J. (2004). Commentary: Promising answers, and the next generation of questions. Psychology, Crime er Law, 10, 335-345.

McGuire, J. (2006). General offending behaviour programmes: Concept, theory, and practice. In C. R. Hollin \& E. J. Palmer (eds), Offending behaviour programmes: Development, application, and controversies (69-111). Chichester, UK: Wiley \& Sons.

Miller, W. R., \& Rollnick, S. (1991). Motivational interviewing: Preparing people to change addictive behavior. Chichester, UK: John Wiley \& Sons.

Nunes, K. L., \& Cortoni, F. (2006). Estimating risk of dropout and expulsion from correctional programs. Research Report $R$-177. Ottawa: Correctional Service Canada.

Ogborne, A. C. (2003). Program Evaluation: An overview of some basic issues. Paper presented at the Correctional Service Canada Addiction Research Centre Summer Institute, août 2003. Montague, Prince Edward Island, Canada.

Ogloff, J. R. P. (1995). Communication de renseignements: les obligations éthiques et juridiques des psychologues qui travaillent en milieu correctionnel. In T. A. Leis, L. L. Motiuk, et J. R. P. Ogloff (eds.), Psychologie médicolégale: Politique et pratiques en milieu correctionnel (15-23). Ottawa: Service correctionnel Canada.

Ogloff, J. R. P., \& Davis, M. R. (2004). Advances in offender assessment and rehabilitation: Contributions of the risk-needs-responsivity approach. Psychology, Crime er Law, 10, 229-242.

Patton, M. Q. (1997). Utilization-focused Evaluation. Thousand Oaks, CA: Sage Publications Inc.

Polaschek, D. L. L. (2006). Violent offender programmes: Concept, theory, and practice. In C. R. Hollin \& E. J. Palmer (eds), Offending behaviour programmes: Development, application, and controversies (113-154). Chichester, UK: Wiley \& Sons.

Polaschek, D. L. L., \& Collie, R. M. (2004). Rehabilitating serious violent adult offenders: An empirical and theoretical stockade. Psychology, Crime and Law, 10, 321-334.

Porteous, N. L., Sheldrick, B. J., \& Stewart, P. J. (2002). Introducing program teams to Logic Models: Facilitating the learning process. Canadian Journal of Program Evaluation, 17, 113-141.

Preston, D. L. (2001). Addressing treatment resistance in corrections. In L.L. Motiuk, \& R. C. Serin (eds.), Compendium 2000 on Effective Corrections (I, 47-55). Ottawa: Correctional Service of Canada.

Prochaska, J. O., \& DiClemente, C. C. (1983). Stages and processes of selfchange of smoking: Toward an integrated model of change. Journal of Consulting and Clinical Psychology, 51, 390-395.

Proulx, J., Brien, T., Ciampi, A., Allaier, J-F., McDonald, M., \& Chouinard, A. (2004). Treatment attrition in sexual aggressors. Paper presented at the $23^{\text {rd }}$ Annual 
Research and Treatment Conference of the Association for the Treatment of Sexual Abusers, octobre 2004. Albuquerque, NM.

Quinsey, V. L. (2008). Seeking enlightenment on the dark side of psychology. Trauma, Violence, and Abuse. 9, 72-83.

Quinsey, V. L., Harris, G. T., Rice, M. E., \& Cormier, C. A. (2006). Violent Offenders: Appraising and Managing Risk ( $2^{\text {nd }}$ ed.). Washington, DC: American Psychological Association.

Quirion, B. (2006). Traiter les délinquants ou contrôler les conduites: le dispositif thérapeutique à l'ère de la nouvelle pénologie. Criminologie, 39 (2), 137-164.

Ward, T., \& Brown, M. (2004). The Good Lives model and conceptual issues in offender rehabilitation. Psychology, Crime $\mathcal{F}$ Law, 10, 243-257.

Ward, T., Howells, K., \& Birgden, A. (2004). The multifactor offender readiness model. Aggression and Violent Behavior, 9, 645-673.

Ward. T., \& Maruna, S. (2007). Rebabilitation: Beyond the risk paradigm. London, UK: Routledge.

Ward, T., Polaschek, D. L. \& Beech, A. R. (2006). Theories of sexual offending. Chichester, U.K.: Wiley.

Welsh, B. C., \& Farrington, D. P. (2001). Evaluating the economic efficiency of correctional intervention programs. In G. A. Bernfeld \& D. P. Farrington (eds.), Offender Rebabilitation in Practice (45-65). Chichester, UK: Wiley.

Wild, T. C., Newton-Taylor, B., \& Alletto, R. (1998). Perceived coercion among clients entering substance abuse treatment: Structural and psychological determinants. Addictive Behaviors, 23, 81-95.

Wong, S. (2000). Psychopathic offenders. In S. Hodgins \& R. Muller-Isbener (eds.), Violence, crime and mentally disordered offenders: Concepts and methods for effective treatment and prevention (87-112). Chichester, UK: John Wiley \& Sons.

Wong, S. \& Hare, R. D. (2005). Guidelines for a psychopathy treatment program. Toronto: Multi-Health Systems.

Wormith, J. S., \& Olver, M. E. (2002). Offender treatment attrition and its relationship with risk, responsivity, and recidivism. Criminal Justice and Behavior, 29, 447-471.

ABSTRACT - In contrast to the Martinson's (1974) 'Nothing works' view, the 'What works?' approach to correctional treatment has focused on whether correctional treatment can be effective in reducing offenders' criminal recidivism. The empirical evidence reviewed in this paper supports that the Andrews and Bonta's (2006) risk-need-responsivity principles provide the bases of an effective model of rehabilitation. Yet, despite great strides toward the development and implementation of evidence-based correctional treatment, there continues to be areas that require further development. In this paper, three issues frequently neglected in offender rehabilitation are additionally discussed, namely the need to attend to motivational issues among offenders, the importance of staff skills and attitude, and the need to continually document and evaluate rehabilitative practices. 
KEYWORDS - Correctional treatment, readiness for treatment, characteristics of therapeutic staff, evidence based.

RESUMEN - Contrariamente a la perspectiva "Nothing works" de Martinson (1974), la orientación "What works?" del tratamiento correccional se ha centrado en la posibilidad de que el tratamiento profesional pueda ser eficaz en la reducción de la reincidencia de los delincuentes. Las pruebas empíricas examinadas en el presente artículo corroboran el hecho de que la aplicación de los principios riesgo-necesidades-receptividad de Andrews y Bonta (2006) proporcionan las bases de un modelo eficaz de rehabilitación. Sin embargo, pese a los grandes progresos realizados en el desarrollo y la puesta en práctica de un tratamiento correccional basado en la evidencia, subsisten áreas que necesitan ser profundizadas. Examinamos también en este artículo tres cuestiones a menudo descuidadas respecto a la rehabilitación del delincuente, a saber, la necesidad de tomar en cuenta las motivaciones de los delincuentes, la importancia de las competencias y actitudes del personal y la necesidad de documentar y evaluar continuamente las prácticas de rehabilitación.

PALABRAS CLAVE - Tratamiento correccional, preparación al cambio, características de los terapeutas, prácticas basadas en la evidencia. 